\title{
Hospital staffs' perceptions of postoperative nutrition among colorectal patients: a qualitative study
}

\begin{abstract}
Background: After lower gastrointestinal surgery, few patients start eating within timeframes outlined by evidence-based guidelines or meet their nutrition requirements in hospital. The present study explored hospital staffs' perceptions of factors influencing timely and adequate feeding after colorectal surgery to inform future interventions for improving postoperative nutritional practices and intakes.
\end{abstract}

Methods: This qualitative exploratory study was conducted at an Australian hospital where Enhanced Recovery After Surgery (ERAS) guidelines had not been formally implemented. One-on-one, semi-structured interviews were conducted with hospital staff who provided care to patients undergoing colorectal surgery. Interviews lasted from 21 to 47 minutes and were audio recorded and transcribed verbatim. Data were analysed using inductive thematic analysis. Emergent themes and sub-themes were discussed by all investigators to ensure consensus of interpretation.

Results: Eighteen staff participated in interviews, including nine doctors, five nurses, two dietitians and two foodservice staff. Staffs' responses formed three themes: (i) variability in perceived acceptability of postoperative feeding; (ii) improving dynamics and communication within the treating team; and (iii) optimising dietary intakes with available resources.

Conclusion: Staff and organizational factors need to be considered when attempting to improve postoperative nutritional among patients who undergo colorectal surgery. Introducing a feeding protocol, enhancing intraprofessional and interdisciplinary communication and 
ensuring the availability of appropriate, nutrient-dense foods are pivotal to improving nutritional practices and intakes.

Key words: Early oral feeding; perioperative care; postoperative care; enhanced recovery after surgery; evidence-based practice; nutrition care

\section{Introduction}

Evidence-based perioperative guidelines recommend that liquid and solid feeding should recommence within 24 hours after colorectal surgery. ${ }^{(1-4)}$ This practice, defined as early oral feeding (EOF), is a safe and beneficial component of the Enhanced Recovery After Surgery (ERAS) program. ${ }^{(5-7)}$ Yet, recent evidence indicates that many patients undergoing postoperative lower gastrointestinal surgery do not recommence nutritionally adequate diets within timeframes outlined by guidelines, nor meet their nutritional requirements while in hospital. ${ }^{(8-12)}$ As such, patients are not exposed to the potential benefits associated with EOF and may incur longer lengths of stay and increased incidence of complications if poor oral intakes persist. ${ }^{(13)}$ Clearly, strategies are required to translate evidence-based nutrition recommendations into habitual practice to improve patient and health care outcomes.

Historically, attempts to implement nutrition guidelines into practice have adopted "one-sizefits-all" approaches, resulting only in small improvements in outcomes. ${ }^{(14-16)}$ The importance of understanding the local context and identifying barriers and enablers to new practice has been recognized as imperative to enacting successful and sustainable change. ${ }^{(17)}$ While previous work has demonstrated that a combination of professional, organizational and patient-related factors influence whether nutrition care practices align with postoperative guidelines, ${ }^{(9,11,13)}$ these findings have come from observational work, limiting our ability to understand these enablers and facilitators at a deeper level. Qualitative methods, including key informant interviews, allow for this in-depth understanding. ${ }^{(18)}$ Few studies have reported staff 
perceptions of barriers and enablers to optimal nutritional care ${ }^{(19)}$ and no studies have exclusively explored this in the context of postoperative colorectal surgery. Understanding the perceptions of those involved in prescribing and delivering postoperative nutrition to patients following colorectal surgery is warranted to understand the barriers and enablers to evidencebased practice.

Provision of nutrition to postoperative colorectal patients involves an interdisciplinary approach. Therefore, having the perspectives of all staff involved in dietary prescription and delivery is important to comprehensively understand factors influencing postoperative nutrition practices. The aim of the present study was to explore dietitians', doctors', nurses' and foodservice staffs' perceptions of providing postoperative nutrition care among colorectal patients. This information will help identify factors that influence timely and adequate feeding after colorectal surgery, and thus inform tailored intervention strategies to improve postoperative nutritional practices and intakes.

\section{Methods}

\section{Study design and setting}

This qualitative study, involving semi-structured interviews, was conducted across a single gastrointestinal surgical ward ( $\sim 28$ beds) in an Australian tertiary teaching hospital. Early Recovery After Surgery (ERAS) guidelines were not formally in use; rather surgeons prescribed feeding in consultation with their team. An electronic foodservice system (EFS) was in operation at the hospital where nursing staff entered patients' dietary prescriptions. This system ensured patients could only order foods appropriate to their prescribed diet. Foodservice staff were responsible for assembling and delivering foods ordered through this system to patients on the ward. Ethical approval was gained by the relevant hospital and university 
Human Research Ethics Committees (reference numbers: HREC/17/QGC/101 and GUREF/2017/389).

\section{Participants and recruitment}

Any full- or part-time dietitian, nurse, doctor or foodservice staff member who provided nutrition care to colorectal patients at the study site was eligible to participate. With assistance from the nurse unit manager and clinical nurse educator, potential participants meeting the inclusion criteria were identified. Maximum variation purposive sampling was used to recruit a mixture of participants in terms of professional role and years' of clinical experience. At least 15 staff members were anticipated to be interviewed based on previous studies of similar design, ${ }^{(20,21)}$ however, recruitment ceased when data saturation was reached (i.e. we conducted interviews until no new information was being offered). This occurred after 16 interviews; however, an additional two staff were subsequently interviewed to confirm that data saturation had, indeed, occurred. Informed consent was obtained from all participants prior to initiating interviews.

\section{Data Collection}

Individual, semi-structured interviews were conducted with all participants. Development of the semi-structured interview guide was an iterative process, informed by theory and data (see supplementary materials). The Theoretical Domains Framework (TDF), a validated framework consisting of 14 domains that has been widely used to help understand human behaviour in complex clinical environments, ${ }^{(22)}$ underpinned the development of the semi-structured interview guide, with two to five questions constructed within each domain. Prior to interviews, questions were further refined based on findings generated from an audit conducted at the site and input from an expert group of clinicians. 
A conversational style of interviewing was adopted with the semi-structured interview guide and staff responses providing direction for the interviewer. All staff were interviewed on-site or over the phone $(\mathrm{n}=1)$ at a time and place convenient to them. Interviews lasted between 21 and 47 minutes (average: 32 minutes), were audio recorded and later transcribed verbatim. The lead researcher (MR), who is trained in interviewing and has a background in dietetics, interviewed all participants between November 2017 and January 2018. Staff demographics such as their gender, years' of clinical experience and position, were recorded on a standardised data collection form.

\section{Data analysis and rigour}

Interview data were thematically analysed using Braun and Clarke's six-step guide to identify emerging themes.(23) Specifically, the lead author (MR) read and reread transcripts for immersion in the data, highlighting key quotes and then developed codes based on participants' verbatim statements. Data from each health profession were analysed separately prior to being combined. Codes were grouped according to similarity into sub-themes, and then themes based on common threads throughout the data. An electronic audit trail was reviewed by a second researcher (SR). Themes and subthemes were interpreted and discussed among all investigators until consensus was reached.

Rigour was upheld using qualitative strategies. ${ }^{(24)}$ The interview guide was pilot tested with two nurses, four dietitians and one surgeon, who provided feedback on the questions and initial insights into the topic. Further, member checking occurred, where the lead author discussed our interpretation of the data with participants who confirmed findings. Reflexivity occurred through the interviewer: i) completing contact summary forms after each interview to enhance future interviews; and ii) documenting their preconceptions and ideas throughout the analysis process to consider how this influenced their approach to interviews and subsequent analysis. 
Frequent discussions among the research team regarding codes, quotes and sub-themes and themes enhanced the credibility and dependability of analysis and findings. Lastly, an audit trail of the analysis process was maintained.

\section{Results}

A total of 18 staff were interviewed, including nine doctors, five nurses, two dietitians and two foodservice staff. Participant demographics are displayed in Table 1 and the medical career structure outlining the description of each medical position is depicted in supplementary file 2 . The majority of participants were female (72\%) and their clinical experience varied from 11 months to 33 years. No staff member that was approached declined to participate. Participant responses formed three themes and various subthemes. These themes, depicted in Table 2 and described below, were identified as the key factors that influenced postoperative nutrition provision following colorectal surgery. (NB: The use of "many" or "most" throughout this section is equivalent to $\sim 75 \%$ or more of the sample mentioned).

\section{(1) Variability in perceived acceptability of postoperative feeding}

All staff spoke about their perceptions regarding the acceptability of early oral feeding and current practice among patients who undergo postoperative colorectal.

\section{Divergent opinions on early solids after surgery}

Overall, many participants expressed positive attitudes towards recommencing feeding after surgery with most recognising the role of nutrition in healing and recovery:

"Nutrition is important. It is like running a marathon and having no nutrition after. Some of our operations take 4 to 6 to 8 hours and we need to provide that nutrition after for their recovery" [P15, Consultant]. 
The majority of participants also recognised that their peers generally held positive views towards recommencing feeding early after surgery. These responses, however, were broadly spoken about in the context of re-commencing fluids (clear or free fluids) in the postoperative period. (NB: The free fluid diet is equivalent to a full liquid diet i.e. includes all foods that are liquid at room temperature such as milk, juice, broth, custard and yoghurt). Staffs' attitudes and beliefs regarding recommencing solids early after surgery varied considerably. Roughly two thirds of the participants expressed concerns about patients re-commencing solids within 24 hours after surgery insisting that there needs to be a balance between safety (i.e. "ensuring everything is ok") and the provision of adequate nutrition. Alternatively, participants who continuously reiterated the concept and importance of ERAS $(\sim 33 \%)$, were 'advocates' for recommencing solids within 24 hours of surgery (NB: these participants had experienced ERAS implementation in other hospitals or were familiar with ERAS literature).

\section{Practice has improved but remains inconsistent}

All staff discussed how the prescription of fluids in the immediate postoperative period was common practice and it would be unusual to see a patient placed nil-by-mouth, unless there was a specific reason why fluids could not be recommenced. Nurses and doctors who had been practicing for more than a decade positively spoke of how this practice had improved from the historic custom of fasting patients until there have been signs of bowel activity. However, most staff described the practice of progressing patients onto solids as highly variable:

"I feel like there is not one set rule. I feel like it is very vast. So some patients will come back and they will be on a clear fluid diet and then they will go to a fluid diet and then they will go to a full diet, once their bowels are opening and with other patients they are ... happy to give, you know, a full diet when they haven't had their bowels open ..." [P10, Enrolled Nurse]. 
Many participants attributed this to variability in surgeon preferences and/or patient-related factors such as age, type of surgery, or incidence/risk of an ileus.

\section{A flexible protocol could help guide practice}

The majority of participants who were 'EOF advocates' thought a protocol would help raise awareness of the "need to provide good nutrition to patients that are recovering". Further, surgeons who were 'EOF advocates' attributed variation and ambiguity in postoperative nutrition care decisions within their unit to a lack of group consensus, and most suggested a protocol could improve practice:

"I think we should have a team approach [to postoperative feeding] rather than an individualised approach to something that is very well described in lots of recent evidence...It should be instituted and it should be instated across the board so that there is consistency and clarity with what postoperative early recovery programs mean" [P16: Fellow].

However, these participants also spoke of the importance of ensuring flexibility, expressing that when protocols are introduced often clinical decision-making is reduced which may lead to comprised patient safety and care:

"The only downside is if we set it as a protocol ... is if you have a patient that has an obvious ileus that is not going to tolerate their oral intake, they may even need a NGT, so if you have someone that is inexperienced and then gives them a meal, they vomit and they aspirate or causes a lot of distress and they do not eat it anyway, so they have no intake at all. That is the only downside I see with it and that is why we like that oversight” [P15: Consultant].

\section{(2) Improving dynamics and communication within the treating team}


All staff spoke about team dynamics and communication and how this can help or hinder timely and adequate nutrition provision.

\section{$\underline{\text { Recognising roles and responsibilities within the team }}$}

Staff described how they and others contributed to postoperative nutrition care within the interdisciplinary team. Surgeons were labelled as the ultimate decision makers in relation to when and what patients ate after surgery. While many doctors acknowledged the role of dietitians in the team, stating that they would seek advice from one if they were concerned about a patient's nutrition, dietitians viewed this dynamic somewhat differently, feeling their recommendations were not necessarily actioned:

"I think that there are certainly surgeons...who really like to take that lead over what the patient is going to have and prefer not to have other people making recommendations...or will accept it but not make any changes" [P04, Dietitian].

Nurses seemed central to many nutrition care processes including screening, assessment and monitoring. In fact, nurses expressed ownership in providing postoperative nutritional care, articulating high intrinsic motivation to "flag", "advocate" and "encourage" oral intakes among "at risk" patients:

"As nurses we need to always be on the ball and make sure our patients are getting the nutrition they need post-op so they don't have long hospital stays and so they can recover well. So we have a duty of care to discuss with the dietitian if we're worried or bring it up with the doctors, you know, be an advocate for the patient" [P05, Registered nurse].

Nurses also conveyed positive attitudes towards the ward dietitians, stating that they were "good at advocating for their patients". Foodservice staff demonstrated high intrinsic 
motivation in delivering nutrition to postoperative patients, stating that they do "everything in our power for that person to get fed".

\section{Overcoming intraprofessional and interdisciplinary hierarchies}

Several staff members, including doctors, nurses and dietitians, spoke about hospital hierarchies and how these could affect postoperative nutritional care. One dietitian stated that protocols to improve perioperative feeding practices had been abandoned in the past as they had been "poo-pooed pretty much by surgeons and anaesthetists". A fellow acknowledged this point by discussing the importance of having surgeons "on-board" when attempting to change practice as they are the "ultimate decision makers" and "can undo the good work of others". In fact, a participant recalled an incident that resulted in a surgeon "swearing and yelling" when asked to comply with a new feeding protocol that the surgeon did not enact. The ways by which intimidation influenced day-to-day nutrition care were also discussed. One nurse stated they "get told what to do" by doctors and that nursing staff may be "scared to take initiative", linking this to disadvantageous outcomes such as "patients getting missed" and thus waiting considerable lengths of time to receive adequate nutrition. Intimidation within the medical team was also identified. A dietitian expressed concerns that surgeons may "intimidate some of the juniors" and as a result, junior staff (i.e. interns and residents) may be "a little afraid to push" the subject with their seniors (i.e. registrar and consultant) regarding upgrading a patient's diet. This was confirmed by an intern who explicitly stated:

"I would hate contacting the registrar...I mean sometimes they would be in theatre (i.e. the operating theatre) so that would mean going down and saying 'can John eat?', which they would be like, 'you know this, we talked about it on ward rounds. I can't believe you interrupted me in theatre. You are expected to know this. My other interns knew this'. Typically what I would do is, if I knew my reg [registrar] was in theatre I 
would be like, I don't know the answer, I will do it safe, so I will keep them on clear fluids until I can ask them in the afternoon" [P13, Intern].

\section{Proficiently communicating nutrition-related information}

The importance of written and verbal communication of dietary orders was raised frequently, particularly among staff whose primary role was to document diet orders (interns and nurses) or staff reliant on accurate and timely diet documentation (dietitians and foodservice staff). Most nurses and both dietitians spoke about issues with vague or incorrect dietary documentation. Staff often attributed this to interns being unfamiliar with the hospital's diet code terminology and with nutrition in general (NB: intern's role within the team was to document the medical team's plans). This was acknowledged by an intern who admitted to being unaware of the hospital's diet code terminologies and to "parroting diet orders" during the early weeks of their rotation. Dietitians discussed the impact of this on patient care, being that written communication was their primary link with senior doctors who were often "torn between wards, clinics, surgeries". If the rationale for continuing patients on certain diets was unclear, it was difficult for dietitians to advocate for the patient. Issues with relaying and updating dietary orders were also discussed. Nursing staff spoke of how other tasks (e.g. bed changes, hematology) could cause delays in updating dietary orders, or if doctors delayed writing their notes and didn't verbally relay diet changes, as it was nurses' responsibility to update dietary orders in electronic foodservice system and on patients' whiteboards. Foodservice staff expressed frustration with delayed dietary documentation (e.g. on patient whiteboards), as it affected their ability to deliver adequate and accurate meals to patients, which they perceived as important:

"Sometimes they [nursing staff] forget to put things on the board and that takes a bit of time [for us to clarify]...we've got to get permission from a nurse, we've got to check with a nurse 
first to see what they are on...we've got to go by what the board says...we don't want to have ask [a nurse] but it's that important to us. We can't give the wrong meal out...I don't mind going and annoying a nurse but sometimes they say they're too busy” [P01: Foodservice staff].

Further, it appeared foodservice largely worked in isolation and that there were "communication issues between us and the nurses".

Despite many staff highlighting problems with communicating dietary changes, most articulated that it was an effective system when each person carried out their task correctly and without considerable delay.

\section{(3) Optimising dietary intakes with available resources}

Staff discussed perceived barriers and enablers to optimising dietary intakes among postoperative patients in the context of their ward environment and resources.

\section{Providing appropriate dietary options}

Many staff discussed the "appropriateness" of meals provided to postoperative patients, often highlighting this as a barrier to optimal nutrition care. However, the definition of 'appropriate' varied across disciplines. Dietitians, nurses and foodservice staff, whose roles were to deliver or oversee nutritional care, raised concerns about the repetitive nature and limited number of dietary choices available on fluid diets. This was thought to contribute to poor oral intakes as patients could get "bored" and may "stop eating" if prescribed these diets for extended periods of time. Some nurses emphasised this as particularly problematic among lactose intolerant patients as there were few lactose-free options on fluid diets. The 'appropriateness' of foods in terms of a patient's chronic condition or clinical status was also raised. Many nurses and a fellow expressed concern with giving diabetic patients clear fluids, recognising the majority of items offered on this diet were carbohydrate rich and thus inappropriate for such patients. 
"The thing is that if they are on a clear fluid diet for too long... the patient basically stops eating because it's a very boring diet. It is basically just jelly and apple juice and black tea or black coffee. Also, there is the issue if somebody is a diabetic; all these jelly and juices are full of sugar. So that really doesn't agree with the diabetics and then the free fluid diet that is another issue when patients are lactose intolerant. Basically there is nothing we can offer. So that's a bit hard" [P08, Clinical nurse].

Alternatively, doctors who spoke about the 'appropriateness' of dietary options did so in the context of foods available on the full diet, suggesting the size and options (e.g. curries and stews) were inappropriate for patients recovering from an ileus, and that lighter, smaller, more frequent meals would be better "tolerated" by patients.

Despite these differences, most staff recognised challenges associated with fluid diets, suggesting they should only be used temporarily ( $<3$ days) given the nutritional needs of surgical patients.

\section{$\underline{\text { Using oral nutrition supplements }}$}

All disciplines were generally accepting of oral nutrition supplements (ONS), with many participants stating they were a useful means of increasing patients' nutritional intakes:

"I get a lot of them [patients] say, I'm really sick of hospital food. I really don't want this and I really don't want that or they are still not feeling up to it. What we can do is use supplementation to help boost their nutritional intake immediately when food may not be giving them enough" [P11, Principal house officer].

However, one nurse and one intern held some reservations, expressing concern that some patients refused ONS simply because of taste (too sweet) or flavour fatigue. Despite participants' overall positive attitudes towards ONS, many nurses stated that short turnaround 
patients (e.g. <1 week) often "get missed" and an intern described patients being prescribed ONS as an "afterthought". There was a stark contrast in the way doctors described using ONS (proactive vs. reactive in treatment for malnutrition). Doctors who were 'ERAS advocates' generally spoke about using ONS proactively, stating it would be beneficial for every patient to receive ONS after bowel surgery. One consultant explicitly stated he was concerned with the costs incurred to the hospital if every patient was prescribed an ONS.

\section{Storing and sourcing foods on the ward}

The need to improve sourcing and storing foods on the ward was a concern raised among nursing and foodservice staff. This was particularly problematic when patients returned to the ward after surgery or had their diet upgraded late in the afternoon, as there were limited resources stored on the ward. Nurses often spoke about actively sourcing foods for patients in these instances, as they recognised the importance of nutrition after surgery; but expressed frustration over the limited storage of foods in close proximity:

"So that's probably...another barrier, like, if a doctor comes round... if they do a late round and say 'they can move from a clear fluid diet to a free fluid diet', if the kitchen is closed we can't give them any food .....[but] we do try and get something if we can and, you know, even if it is jelly or yogurt or whatever from the fridge...I mean if someone is starving we will try and find them something" [P06, Team leader nurse].

While nursing staff articulated high intrinsic motivation for sourcing foods, two discussed how they may delay delivering foods to patients (despite having a diet prescribed) upon returning to the ward after surgery in fear of adverse outcomes (e.g. vomiting or aspiration).

\section{Discussion}


This study aimed to explore hospital staffs' perceptions of nutrition care among patients who have undergone colorectal surgery. Team dynamics/communication, staff attitudes towards postoperative nutrition, and dietary resources available at the hospital were identified as key factors influencing timely and adequate postoperative feeding. This information will help inform tailored intervention strategies to improve postoperative nutrition care.

Overall, the majority of staff expressed positive attitudes towards the role of nutrition in healing and recovery after surgery. Yet, staff recognized that patients were often commenced on solid diets several days after surgery and ate poorly whilst in hospital; findings consistent with

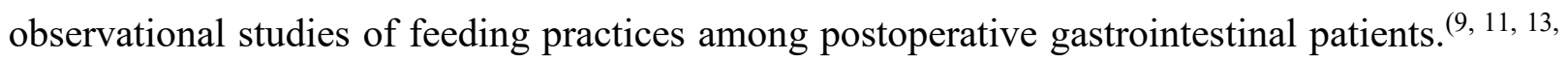
25) Many staff attributed variability in the prescription of solids to differences in surgeons' preferences and patient-related factors, such as the incidence or risk of postoperative ileus. Staff who were 'advocates' for EOF discussed the idea of introducing a protocol to facilitate an evidence-based team approach towards postoperative feeding, given the variability in surgeons' practices. An environment which includes policies and encourages a social or cultural approach to behavior change has the potential to facilitate desired change. ${ }^{(26)}$ As identified in the current study, consultation with all key stakeholders, especially surgeons who were seen as the ultimate decision makers in determining diet upgrades, would be required to successfully develop and embed a feeding protocol within habitual practice; which is consistent with implementation literature. ${ }^{(27)}$ An important idea that emerged during interviews was the need for flexibility within a postoperative feeding protocol to facilitate the use of clinical judgment and ensure safe and patient-centred care. This aligns with previous work on the introduction of evidencebased treatments where clinicians were concerned with reduced opportunities to exercise clinical judgment and protocols not being flexible enough for complex cases (rather than having negative attitudes toward the innovation itself). ${ }^{(28-30)}$ Further, patients' perceive ERAS protocols as being inflexible and would prefer less rigid guidelines. ${ }^{(31,32)}$ Therefore, introducing 
a feeding protocol that allows flexibility for clinicians to exercise their clinical judgement, and factor in patients' preferences and needs, may facilitate a consistent and positive culture towards postoperative nutrition practices.

Breakdowns and delays in verbal and written communication of nutrition decisions within the interdisciplinary team were common areas of improvement raised by staff; a finding congruent with work exploring barriers and facilitators to ERAS compliance. ${ }^{(12,28)}$ Delays were recognised to occur throughout the nutrition care system, from the medical team upgrading patients' diets at the bedside to foodservice staff delivering meals. Communication breakdowns in nutrition care have previously been attributed to disciplines working in individual silos, ${ }^{(19)}$ which to an extent, were demonstrated within our findings, particularly in regards to food delivery. Further, the accuracy and comprehensive of dietary documentation were raised as concerns by nurses and dietitians, who were heavily reliant on this information to undertake their roles and responsibilities in nutrition delivery. These issues were attributed to unfamiliarity with general nutrition among junior doctors, whose role was to document the treating team's plans. Indeed, this was confirmed by interns in the current study and is consistent with previous work describing the need to advance nutrition education among doctors. $^{(33,34)}$ Yet, the importance of including nutrition in the training of medical students, as well as in the continuing education of practicing clinicians, remains a low priority. ${ }^{(35)}$ These findings support the need for further integration of nutrition into education and training programs for doctors to improve knowledge. Given the highly co-ordinated nature of postoperative care, clear channels of communication need to be embedded into routine clinical practice to improve the delivery of timely and adequate nutritional care.

The overall social climate and team dynamics were discussed by staff and appeared to directly influence nutrition care. Hospital systems consist of hierarchical structures, whereby senior doctors are the ultimate decision-makers within the treating team. ${ }^{(36)}$ While hierarchical 
systems facilitate order and help organise patient goals, when intimidation and humiliation exist within a team, it can create stressful environments for staff where they may feel undervalued and patient care can be comprised. ${ }^{(36,37)}$ In the current study, one intern recalled a specific experience whereby the fear of clarifying a diet order with her senior led to prolonged inadequate nutrition for a patient. Further, several staff spoke of how they thought junior doctors and non-medical staff may be intimated to raise nutritional concerns with more senior doctors, resulting in patients receiving inadequate nutrition for excessive periods of time. These findings emphasise the need to look beyond raising awareness and increasing knowledge among staff when attempting to improve postoperative nutrition care. Clinical environments where good internal and external dialogue takes place and individuals feel supported have fewer stressed team members and provide better health care. ${ }^{(38,39)}$ Developing interpersonal relationships have been identified as a key characteristic for overcoming intimidation and rigid hierarchical structures in the clinical setting. ${ }^{(36,40)}$ As such, efforts should be directed towards building strong intraprofessional and interdisciplinary relationships to facilitate optimal postoperative nutrition care.

Dietary resources were identified as another key factor influencing adequate and timely postoperative nutrition. Nursing staff raised concerns about limited access to readily available foods, which was a problem if patients were admitted to the ward in the evening when the kitchen was closed. This finding is congruent with an observational study undertaken at the study site, reporting highly variable median time differences (1-9 hours) in the delivery of patients' first meal after surgery. ${ }^{(9)}$ Further, while several staff described using ONS to support patient intake, which has been shown to improve nutrition intakes ${ }^{(41-43)}$ and reduce length of stay $^{(43)}$, nurses and interns disclosed that ONS were not routinely prescribed to patients who underwent uncomplicated bowel surgery; a finding likely attributed to differences in staffs' perceptions of using ONS (reactive vs proactive treatment). Lastly, the palatability, meal 
size/options, nutritional content, and/or repetitive nature of dietary items offered on the liquid and solid diets were raised as factors contributing to poor oral intakes among patients. Indeed, this is consistent with previous studies investigating nutritional provision and intakes among patients prescribed liquid diets ${ }^{(11,25)}$ and previous work undertaken at the study site exploring postsurgical patients' food preferences and perceptions. ${ }^{(44)}$ Collectively, these results indicate that hospitals need to ensure postsurgical patients have access to a variety of appropriate, adequate and timely foods and that patients and staff are aware of the dietary options available to them.

A clear limitation of the current study is that the generalisability of the findings may be limited to the participants and setting studied. Despite using maximum variation purposive sampling and continuing interviews until data saturation was reached, it is possible that some views are not represented in our sample. A strength of the present study is we have addressed a number of practical recommendations identified by clinicians, which if addressed, could enhance postoperative feeding practices and intakes among colorectal patients (Box 1).

\section{Conclusion}

Staff and organisational factors need to be considered when attempting to improve postoperative nutrition. Introducing a feeding protocol, enhancing intraprofessional and interdisciplinary communication and ensuring appropriate nutrient-dense foods are readily available are pivotal to improving nutrition care practices and dietary intakes among patients who undergo colorectal surgery. Future work should consider assessing the efficacy of and processes underpinning a multifaceted intervention incorporating strategies to address these factors. 


\section{References}

1. Nygren J, Thacker J, Carli F, Fearon KCH, Norderval S, Lobo DN, et al. Guidelines for Perioperative Care in Elective Rectal/Pelvic Surgery: Enhanced Recovery After Surgery (ERAS ${ }^{\circledR}$ ) Society Recommendations. World J Surg. 2013;37(2):285-305.

2. Gustafsson UO, Scott MJ, Schwenk W, Demartines N, Roulin D, Francis N, et al. Guidelines for Perioperative Care in Elective Colonic Surgery: Enhanced Recovery After Surgery (ERAS ${ }^{\circledR}$ ) Society Recommendations. World J Surg. 2013;37(2):259-84.

3. Weimann A, Braga M, Harsanyi L, Laviano A, Ljungqvist O, Soeters P, et al. ESPEN Guidelines on Enteral Nutrition: Surgery including Organ Transplantation. Clin nutr. 2006;25(2):224-44.

4. Wischmeyer PE, Carli F, Evans DC, Guilbert S, Kozar R, Pryor A, et al. American Society for Enhanced Recovery and Perioperative Quality Initiative Joint Consensus Statement on Nutrition Screening and Therapy Within a Surgical Enhanced Recovery Pathway. Anesth Analg. 2018;126(6):1883-95.

5. Zhuang $C, Y e X$, Zhang C, Dong $Q$, Chen B, Yu Z. Early versus traditional postoperative oral feeding in patients undergoing elective colorectal surgery: a meta-analysis of randomized clinical trials. Dig Surg. 2013;30(3):225-32.

6. Osland E, Yunus R, Khan S, Memon M. Early Versus Traditional Postoperative Feeding in Patients Undergoing Resectional Gastrointestinal Surgery: A Meta-Analysis. JPEN. 2011;35(4):473-87.

7. Andersen HK, Lewis SJ, Thomas S. Early enteral nutrition within $24 \mathrm{~h}$ of colorectal surgery versus later commencement of feeding for postoperative complications. Cochrane Database of Syst Rev. 2006;1(4).

8. Rattray M, Roberts S, Marshall A, Desbrow B. A systematic review of feeding practices among postoperative patients: is practice in-line with evidenced-based guidelines? J Hum Nutr. 2017;31(2):151-67.

9. Rattray $M$, Marshall $A$, Desbrow $B$, Roberts $S$. Feeding practices and nutritional intakes among non-critically ill, postoperative adult patients: An observational study. Nutr Clin Pract. 2018; doi: 10.1002/ncp.10103.

10. Byrnes A, Banks M, Mudge A, Young A, Bauer J. Enhanced Recovery After Surgery as an auditing framework for identifying improvements to perioperative nutrition care of older surgical patients. Eur J Clin Nutr. 2017;72(6):913-6.

11. Byrnes A, Worrall J, Young A, Mudge A, Banks M, Bauer J. Early post-operative diet upgrade in older patients may improve energy and protein intake but patients still eat poorly: an observational pilot study. J Hum Nutr Diet 2018; doi: 10.1111/jhn.12572.

12. Robertson TR, Eldridge NE, Rattray ME, Roberts SJ, Desbrow B, Marshall AP, et al. Early oral feeding after colorectal surgery: A mixed methods study of knowledge translation. Nutr Diet. 2018;75(4):345-52.

13. Garth A, Newsome C, Simmance N, Crowe T. Nutritional status, nutrition practices and postoperative complications in patients with gastrointestinal cancer. J Hum Nutr. 2010;23(4):393-401.

14. Doig GS, Simpson F, Finfer S, Delaney A, Davies AR, Mitchell I, et al. Effect of Evidence-Based Feeding Guidelines on Mortality of Critically III Adults: A Cluster Randomized Controlled Trial. JAMA. 2008;300(23):2731-41.

15. Jain MK, Heyland D, Dhaliwal R, Day AG, Drover J, Keefe L, et al. Dissemination of the Canadian clinical practice guidelines for nutrition support: Results of a cluster randomized controlled trial. Crit Care Med. 2006;34(9):2362-9.

16. Martin CM, Doig GS, Heyland DK, Morrison T, Sibbald WJ, Southwestern Ontario Critical Care Research N. Multicentre, cluster-randomized clinical trial of algorithms for critical-care enteral and parenteral therapy (ACCEPT). CMAJ. 2004;170(2):197-204.

17. Cochrane LJ, Olson CA, Murray S, Dupuis M, Tooman T, Hayes S. Gaps between knowing and doing: Understanding and assessing the barriers to optimal health care. J Contin Educ Health Prof. 2007;27(2):94-102. 
18. Miles M, Huberman A, Saldana J. Qualitative data analysis: a methods sourcebook. 3rd ed. Arizona State University, USA: Sage Publishing; 2014.

19. Laur C, Valaitis R, Bell J, Keller H. Changing nutrition care practices in hospital: a thematic analysis of hospital staff perspectives. BMC Health Serv Res. 2017;17(1):498-513.

20. Patey AM, Islam R, Francis JJ, Bryson GL, Grimshaw JM, Driedger M, et al. Anesthesiologists' and surgeons' perceptions about routine pre-operative testing in low-risk patients: Application of the Theoretical Domains Framework (TDF) to identify factors that influence physicians' decisions to order pre-operative tests. Implement Sci. 2012;7(1):52-65.

21. Roberts S, Marshall A, Chaboyer W. Hospital staffs' perceptions of an electronic program to engage patients in nutrition care at the bedside: a qualitative study. BMC Med Inform Decis Mak. 2017;17(1):105-16.

22. Cane J, O'Connor D, Michie S. Validation of the theoretical domains framework for use in behaviour change and implementation research. Implement Sci 2012;7(1):37.

23. Braun V, Clarke V. Using thematic analysis in psychology. Qual Res Psychol. 2006;3(2):77-101.

24. Lincoln Y, Guba E. Naturalistic Inquiry. United States of America: SAG Publications 1985.

25. Rattray $M$, Desbrow $B$, Roberts $S$. Comparing nutritional requirements, provision and intakes among patients prescribed therapeutic diets in hospital: an observational study. Nutr. 2017;39-40:506.

26. Weiner BJ, Belden CM, Bergmire DM, Johnston $\mathrm{M}$. The meaning and measurement of implementation climate. Implement Sci. 2011;6(1):78-90.

27. Straus SE, Tetroe J, Graham ID, ebrary I. Knowledge translation in health care: moving from evidence to practice. Chichester, West Sussex: John Wiley \& Sons; 2013.

28. Lyon A, Solomon MJ, Harrison JD. A Qualitative Study Assessing the Barriers to Implementation of Enhanced Recovery After Surgery. World J Surg. 2014;38(6):1374-80.

29. Weisz JR, Borntrager CF, Chorpita BF, Higa-McMillan C. Provider Attitudes Toward EvidenceBased Practices: Are the Concerns With the Evidence or With the Manuals? Psychiatr Serv. 2009;60(5):677-81.

30. Rattray M, Roberts S, Desbrow B, Wullschleger M, Robertson T, Hickman I, et al. A qualitative exploration of factors influencing medical staffs' decision-making around nutrition prescription after colorectal surgery. BMC Health Serv Res. 2019; In press.

31. Gillis C, Gill M, Marlett N, MacKean G, GermAnn K, Gilmour L, et al. Patients as partners in Enhanced Recovery After Surgery: A qualitative patient-led study. BMJ Open. 2017;7(6):e017002.

32. Sibbern T, Bull Sellevold V, Steindal SA, Dale C, Watt-Watson J, Dihle A. Patients' experiences of enhanced recovery after surgery: a systematic review of qualitative studies. J Clin Nurs. 2017;26(910):1172-88.

33. Raman M, Violato C, Coderre S. How Much do Gastroenterology Fellows Know About Nutrition? J Clin Gastroenterol. 2009;43(6):559-64.

34. Vetter ML, Herring SJ, Sood M, Shah NR, Kalet AL. What Do Resident Physicians Know about Nutrition? An Evaluation of Attitudes, Self-Perceived Proficiency and Knowledge. J Am Coll Nutr. 2008;27(2):287-98.

35. Kris-Etherton PM, Akabas SR, Bales CW, Bistrian B, Braun L, Edwards MS, et al. The need to advance nutrition education in the training of health care professionals and recommended research to evaluate implementation and effectiveness. Am J Clin Nutr. 2014;99(5):1153S-66S.

36. Crowe S, Clarke N, Brugha R. 'You do not cross them': Hierarchy and emotion in doctors' narratives of power relations in specialist training. Soc Sci Med. 2017;186:70-7.

37. Vaughn VM, Saint S, Krein SL, Forman JH, Meddings J, Ameling J, et al. Characteristics of healthcare organisations struggling to improve quality: results from a systematic review of qualitative studies. BMJ Qual Saf. 2018;28(1):74-84.

38. Firth-Cozens J. Organisational trust: The keystone to patient safety. Qual Saf Health Care. 2004;13(1):56-61.

39. Young GJ, Charns MP, Daley J, Forbes MG, Henderson W, Khuri SF. Best Practices for Managing Surgical Services: The Role of Coordination. Health Care Manage Rev. 1997;22(4):72-81. 
40. Engel J, Prentice D, Taplay K. A Power Experience: A Phenomenological Study of Interprofessional Education. J Prof Nurs. 2017;33(3):204-11.

41. Basse L, Jakobsen DH, Billesbølle $\mathrm{P}$, Werner $\mathrm{M}$, Kehlet $\mathrm{H}$. A clinical pathway to accelerate recovery after colonic resection. Ann Surg. 2000;232(1):51-7.

42. Henriksen MG, Hansen HV, Hessov I. Early oral nutrition after elective colorectal surgery: influence of balanced analgesia and enforced mobilization. Nutrition. 2002;18(3):263-7.

43. Yeung SE, Hilkewich L, Gillis C, Fenton TR, Heine JA. Protein intakes are associated with reduced length of stay: a comparison between Enhanced Recovery After Surgery (ERAS) and conventional care after elective colorectal surgery. Am J Clin Nutr. 2017;106(1):44-51.

44. Rattray M, Marshall A, Desbrow B, Roberts S. A qualitative exploration of patients' experiences with and perceptions of recommencing feeding after colorectal surgery. J Hum Nutr Diet. 2018; doi: 10.1111/jhn.12596. 